\title{
Coupling and Loading Measurements for a Dielectric- Filled ICRF Waveguide Coupler
}

\author{
J. L. LEE, J. E. SCHARER, AND B. M. JOST
}

\begin{abstract}
Experiments were carried out to verify models of the reflection coefficients and the phase transitions for matched or loaded dielectric-filled waveguide couplers in the ion cyclotron range of frequencies (ICRF). A model for the plasma load simulation in the waveguide is developed and examined experimentally using the time-domain features of an HP 8510 network analyzer. The loss properties of deionized water, which was used for laboratory waveguide dielectric tests, are also discussed. Close agreement between the coupling models and measurements is obtained, indicating its attractiveness for ICRF coupling.
\end{abstract}

\section{INTRODUCTION}

$\mathrm{M}$ ANY ANTENNAS have been analyzed and constructed and experiments have been carried out with them for coupling high power fast magnetosonic waves into plasmas in the ion cyclotron range of frequencies (ICRF, 30-200 MHz). This is done to heat a confined plasma in current tokamaks and for future tokamak reactors to reach fusion breakeven and ignition temperatures. The coupling antennas considered so far include coil antennas [1]-[3], resonant cavities [4], and cutoff waveguide couplers [5]. Coil antennas have been moderately successful in coupling average powers of up to $2 \mathrm{MW}$ per antenna [1] and average power fluxes of $0.5-2 \mathrm{~kW} / \mathrm{cm}^{2}$ [6]. On the other hand, waveguide antennas have been used successfully in coupling average powers of up to 1 MW with an average power flux of $10 \mathrm{~kW} / \mathrm{cm}^{2}$ during lower hybrid heating [7]. For higher power, structural rigidity, reactor hardened configurations, and the possibility of coupling without a Faraday shield in a large tokamak, waveguide couplers offer a viable alternative to coil antennas.

We have previously analyzed a shorted-probe feeding scheme and its coupling, tuning, and loading properties [8]. In this paper, we present a series of measurements that we have carried out for the matching and simulatedplasma loading of our dielectric-filled ICRF waveguide coupler with a comparison to our analysis.

For convenience, the results of our coupling analysis [8] are given as follows: When the waveguide coupler is matched at the output (plasma) port, port 2 in Fig. 1, the input field reflection coefficient at port 1 is

Manuscript received February 29, 1988; revised August 16, 1988. This work was supported by the Department of Energy under Grant DEFG0286ER52133.

The authors are with the Department of Electrical and Computer Engineering, University of Wisconsin-Madison, Madison, WI 53706.

IEEE Log Number 8824366.

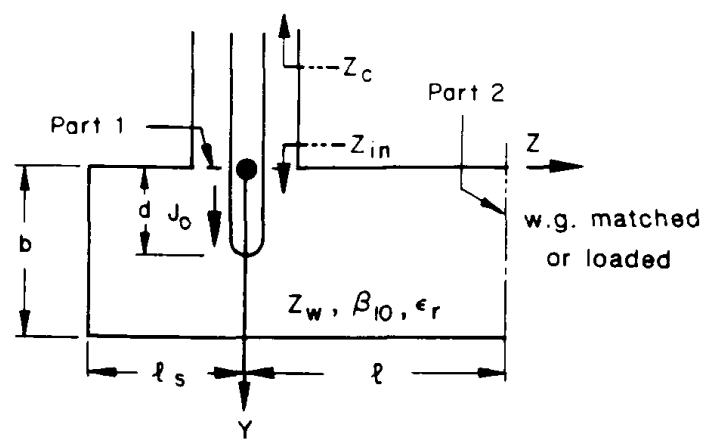

(a)

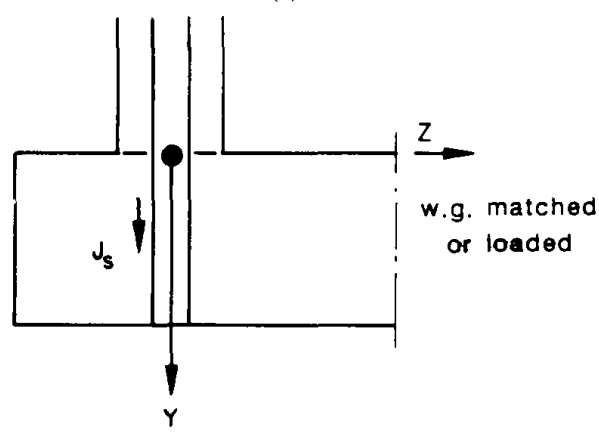

(b)

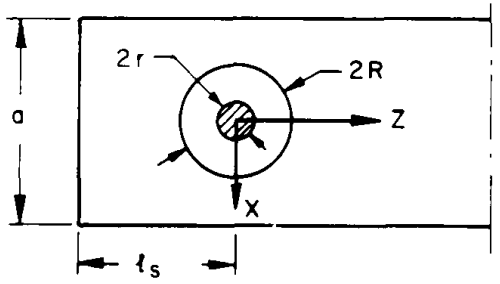

(c)

Fig. 1. Coaxial feeds for the waveguide: (a) Open-ended probe; (b) Shorted probe; and (c) Top view of the feeds.

$$
S_{11}=\frac{\left(R_{\mathrm{in}}-R_{0}\right)+j X_{\mathrm{in}}}{\left(R_{\mathrm{in}}+R_{0}\right)+j X_{\mathrm{in}}}
$$

where $R_{0}$ is the characteristic impedance of the coaxial line, and the corresponding VSWR and power coupling efficiency are

$$
V S W R=\frac{1+\left|S_{11}\right|}{1-\left|S_{11}\right|}
$$


and

$$
\left|S_{21}\right|^{2}=1-\left|S_{11}\right|^{2}
$$

respectively. The coupler input impedance with a shorted probe, $Z_{\text {in }}=R_{\text {in }}+j X_{\text {in }}$, can be obtained as [8]

$$
\begin{aligned}
R_{\mathrm{in}}= & \frac{2 \eta_{\epsilon} \tan ^{2}\left(k_{0 \epsilon} d\right)}{a b \beta_{10} k_{0 \epsilon}} \sin ^{2}\left(\beta_{10} l_{s}\right) \\
X_{\mathrm{in}}= & \frac{\eta_{\epsilon} \tan ^{2}\left(k_{0 \epsilon} d\right)}{2 \pi b k_{0 \epsilon}}\left(\left\{\frac{2 \pi}{a \beta_{10}}\right\} \sin \left(2 \beta_{10} l_{s}\right)+\ln \left\{\frac{2 a}{\pi r}\right\}\right. \\
& +\frac{0.0518 k_{0 \epsilon}^{2} a^{2}}{\pi^{2}} \\
& \left.-2\left(1-\frac{2 r}{a}\right)-2 k_{0 \epsilon}^{2} \sum_{n=1}^{\infty} \frac{K_{0}\left(k_{n} r\right)}{k_{n}^{2}}\right)
\end{aligned}
$$

where

$$
\begin{aligned}
& a, b \quad \text { Waveguide width and height. } \\
& r, d \quad \text { Probe radius and depth. } \\
& l_{s} \quad \text { Tuning distance between the probe and the } \\
& \text { sliding-short. } \\
& \eta_{\epsilon} \quad \eta_{0} / \sqrt{\epsilon_{r}}=\text { intrinsic impedance of the dielec- } \\
& \beta_{10} \quad\left[k_{0 \epsilon}^{2}-(\pi / a)^{2}\right]^{1 / 2}=T E_{10} \text { mode propagation } \\
& \text { constant. } \\
& k_{n} \quad\left[(n \pi / b)^{2}-k_{0 \epsilon}^{2}\right]^{1 / 2} \text {. } \\
& K_{0}\left(k_{n} r\right) \text { Modified zero-order Bessel function of the } \\
& \text { second kind. }
\end{aligned}
$$

Note that we have used a shorted probe so that $b=d$ in (2a) and (2b).

When we terminate the coupler's output port with a plasma or a "simulated" plasma (in terms of the same plasma surface impedance), we can calculate the new input field reflection coefficient at port 1 as

$$
S_{11}^{\prime}=\frac{S_{11}+\Gamma^{\prime}}{1+S_{11}^{*} \Gamma^{\prime}}
$$

where $\Gamma^{\prime}=\Gamma e^{j 2 \phi_{12}}, \Gamma$ is the reflection due to the plasma load at the coupling aperture, and $\phi_{12}$ is the phase angle of the transmission coefficient $S_{12}$. The corresponding VSWR and power coupling efficiency can again be calculated using (1b) and (1c), respectively, with $\left|S_{11}\right|$ replaced by $\left|S_{11}^{\prime}\right|$. Since $\phi_{12}$ is the difference between the phase at the input coax terminal plane and that at the waveguide aperture, it is called the phase transition and is expressed in terms of the waveguide parameters as

$$
\phi_{12}=-\left[\frac{\pi}{2}+\beta_{10}\left(l+l_{s}\right)+\phi_{\text {in }}\right] \text {. }
$$

The distances $l$ and $l_{s}$ are shown in Fig. 1 . The phase angle of the input impedance $Z_{\text {in }}$ is $\phi_{\text {in }}=\tan ^{-1}\left(X_{\text {in }} / R_{\text {in }}\right)$ and $\beta_{10}$ is the propagation constant defined for the $T E_{10}$ mode of the waveguide.

Equations (1)-(4) together form a complete equation set to compute the input reflection coefficient $S_{11}^{\prime}$, VSWR, or power coupling efficiency of the plasma loaded waveguide coupler once $\Gamma, Z_{\text {in }}$, and the waveguide parameters can be determined. We proceed to verify the accuracy of these equations by fabrication of an ICRF waveguide coupler and measurements of its reflection coefficient for different load impedances. The following sections describe our laboratory measurements to examine $\phi_{12}$ for the phase shift of the transition, $S_{11}$ for a matched waveguide, $S_{11}^{\prime}$ for a plasma loaded waveguide and how we produced a simulated plasma load in a waveguide.

\section{Phase Transition and $Q$ Values}

The wave coupling property determined by (3) for a plasma loaded waveguide coupler depends heavily on the accuracy of the phase shift $\phi_{12}$ from the viewpoint of tuning. Phase-accurate calibration standards were unavailable for the full-scale waveguide tests; therefore, we first experimentally verified the phase shift, $\phi_{12}$, which is governed by (4), in a scaled model. Measurements were made for an $X$-band (8-12 GHz) coax-waveguide transition with a waveguide width of $2.278 \mathrm{~cm}$ and a probe radius of $0.119 \mathrm{~cm}$. The transmission phase delay was measured using an HP 8410 network analyzer for a coax-waveguide-waveguide-coax transition as shown in Fig. 2.

Fig. 3 shows the $\phi_{12}$ values versus the normalized frequency $f / f_{c}$. where $f_{c}$ is the cutoff frequency for the $T E_{10}$ mode. The comparison of the two curves indicates that the agreement between the experiment and our theory (4) is very good, as shown by the small deviation over the range $1.29<f / f_{c}<1.9$. The discrepancy between the two curves at the lower frequency end may result from the error involved in $\phi_{\text {in }}$, the phase angle of the coupler input impedance. We have assumed a surface current of a sinusoidal profile along the feed probe (Fig. 1(b)) and of an azimuthal symmetry (Fig. 1(c)) around the probe. Jarem [9] has indicated that these assumptions may introduce some errors for large radii probes.

Since our waveguide coupler is dielectric-filled, the loss property of the dielectric filler is a concern for our laboratory tests. Deionized water was chosen for our waveguide couplers as the dielectric filler because it is low in cost and deforms continuously around the sliding-short section, thus allowing tunability. Most importantly, it has a very high permittivity in the ICRF $\left(\epsilon_{r}=78\right)$ which enables us to design a compact waveguide at a low frequency.

The resonant cavity method was applied and both loop and probe excitations were used to test the $Q$ values of the deionized water at $100 \mathrm{MHz}$. The average of the measured $Q$ values is 185 , which leads to a power attenuation of $0.13 \mathrm{~dB} / \mathrm{ft}$ at $100 \mathrm{MHz}$. This small power attenuation level indicates that deionized water in the ICRF is acceptable as an adjustable dielectric filler to test our design formulas, since our dielectric-filled waveguide coupler would not be longer than five feet. Comparison between our measured $0.13 \mathrm{~dB} / \mathrm{ft}$ attenuation and that presented by Jackson [10] shows excellent agreement. 


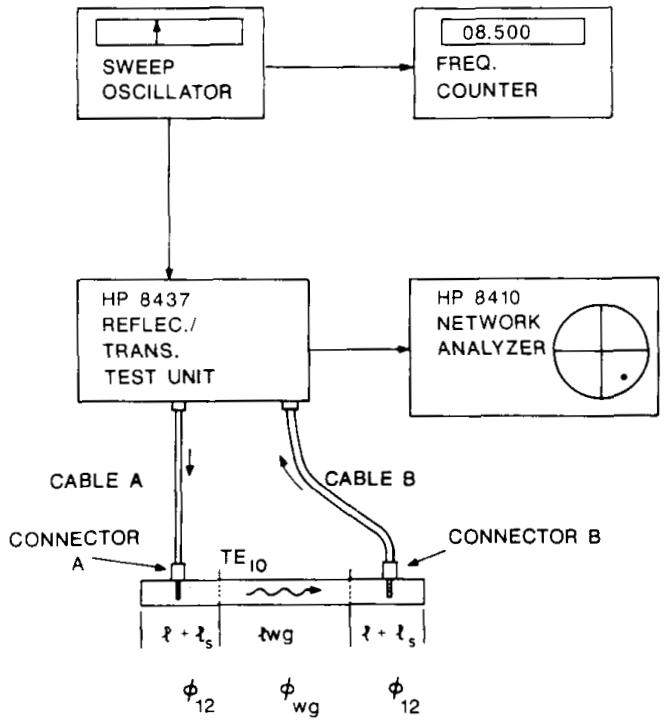

Fig. 2. Measurement set-up for the phase shift $\phi_{12}$.

$\mathrm{f}(\mathrm{GHz})$

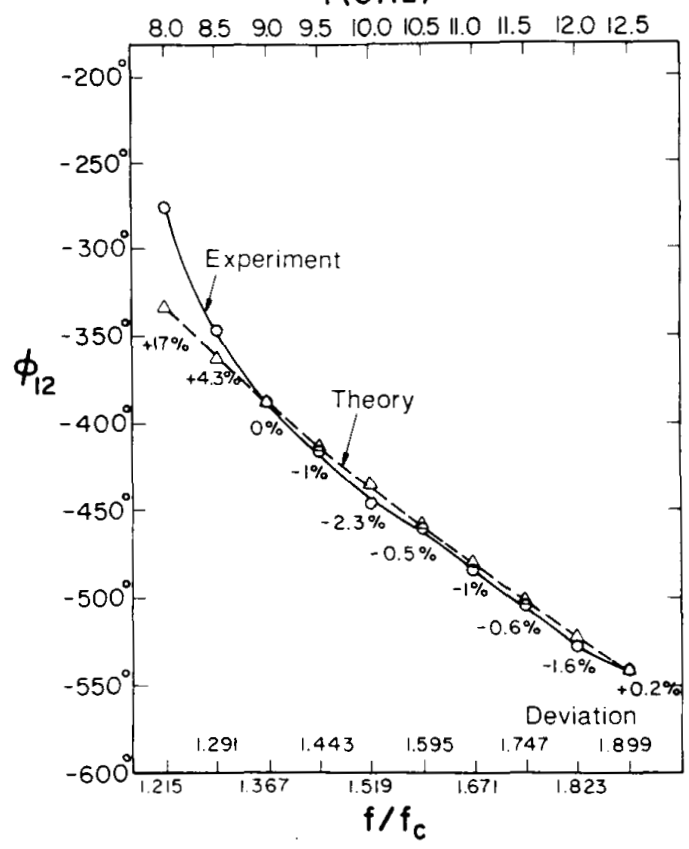

Fig. 3. Comparison of the phase shift between theory and experiment.

\section{Matched Waveguide Coupler}

We have constructed a sliding-short tunable $24.8 \mathrm{~cm} \times$ $12.4 \mathrm{~cm}$ rectangular waveguide coupler driven by shorted coaxial probes of selected radii; this waveguide system was used throughout the remainder of our measurements. The lack of commercially matched loads for our waveguide has led us to construct a tapered matched load using VF-60 microwave absorbers (Emerson and Cuming). Prior to measurements with the dielectric-filled waveguide coupler, the matched load, as shown in Fig. 4, was

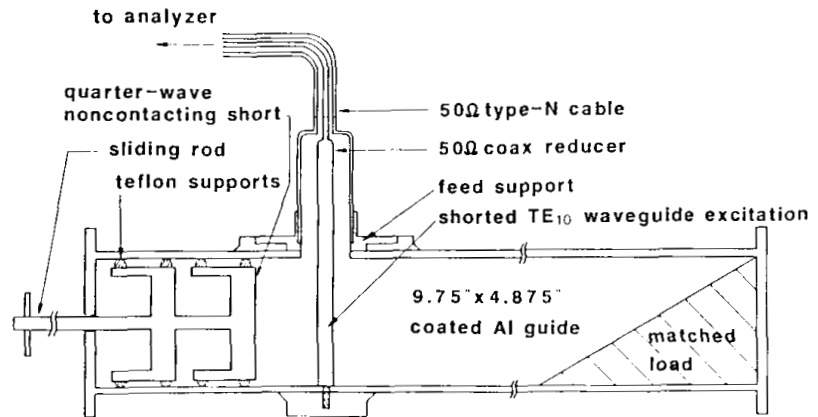

Fig. 4. Side view of the tunable waveguide coupler. Dielectric level is at the junction of the coax and the waveguide (waveguide internal size: $24.8 \times 12.4 \mathrm{~cm}$ ).

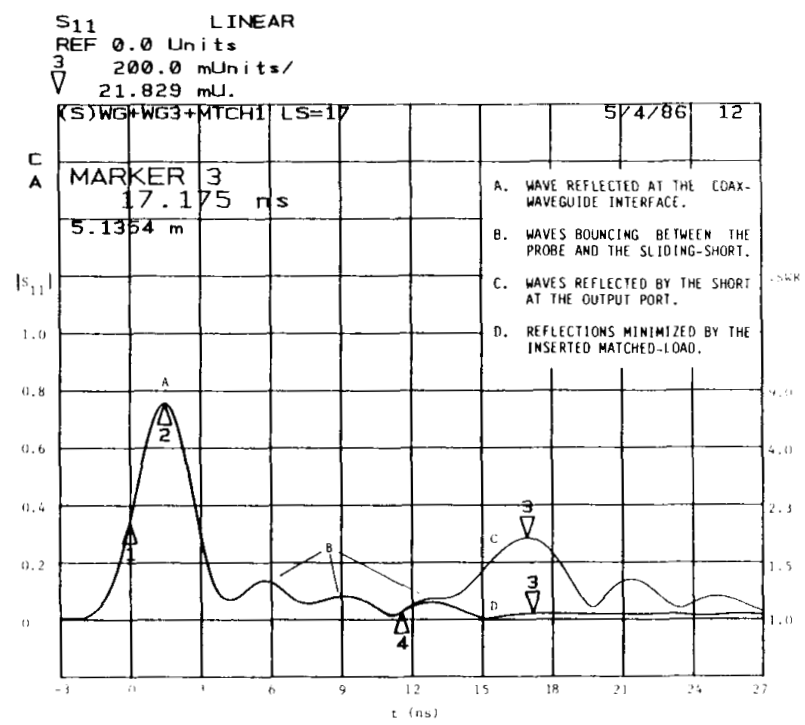

Fig. 5. Time-domain response of $\left|S_{11}\right|$ when the output port of the airfilled waveguide is shorted (upper) and matched (lower), respectively.

tested to examine its absorption properties using the timedomain gating functions of an HP 8510 network analyzer (which generates RF burst signals for band-limited test devices such as waveguides) [11], [12]. Fig. 5 shows the time-domain response of $\left|S_{11}\right|$ of the waveguide coupler before and after the matched load was inserted. The "gating window" of the analyzer was then activated to isolate the response due to the matched load, as presented in Fig. 5 , and the Fourier transform operation of the network analyzer was used to obtain its frequency response, i.e., $\left|S_{11}\right|$ versus frequency. The absorption of the matched load turns out to be more than $20 \mathrm{~dB}$ (VSWR $\leq 1.22$ ) over both the RF ( $\simeq 750 \mathrm{MHz}$, air-filled) and ICRF $(\simeq 85$ $\mathrm{MHz}$, dielectric-filled) bands. These absorption measurements show that our large tapered matched load is adequate for use in the input reflection measurements for the matched waveguide coupler.

We next measured the input reflection coefficient, $S_{11}$, for the matched, dielectric-filled waveguide coupler. The measurement setup is shown in Fig. 4 in which the aforementioned tapered matched load has been utilized. The 


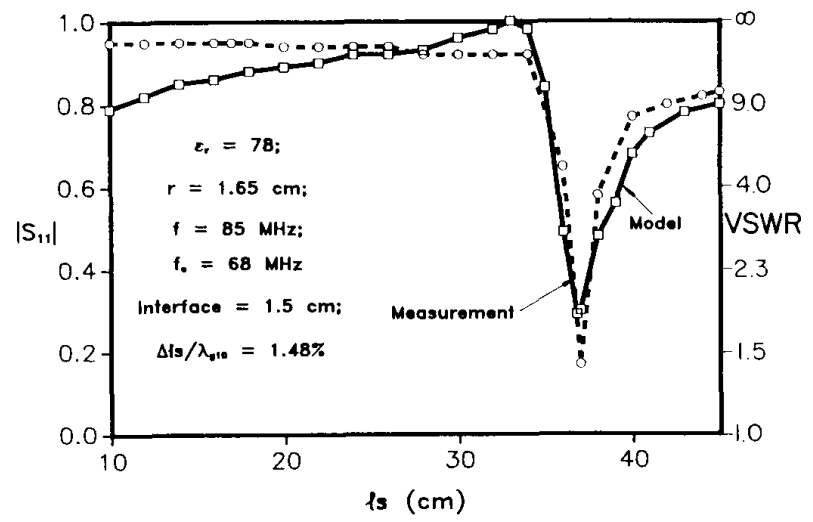

Fig. 6. Field reflection versus the sliding-short tuning distance for a matched, dielectric-filled waveguide coupler $(r=1.65 \mathrm{~cm})$

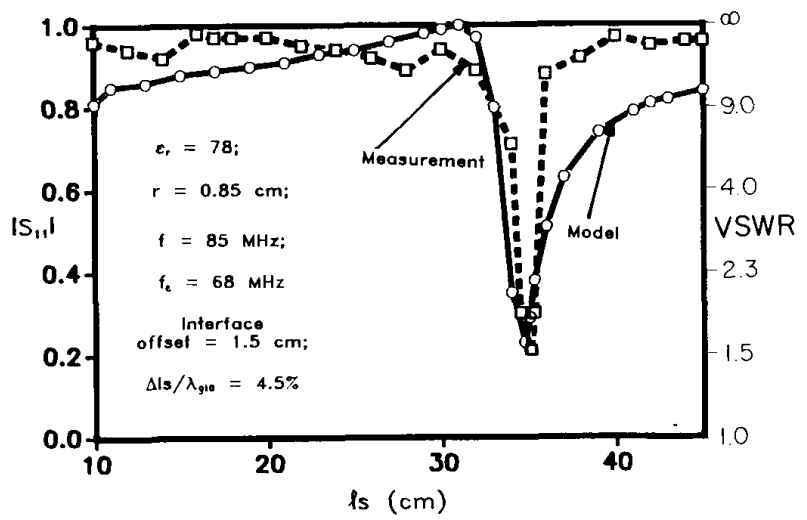

Fig. 7. Field reflection versus the sliding-short tuning distance for a matched, dielectric-filled waveguide coupler $(r=0.85 \mathrm{~cm})$

backside sliding-short is adjusted to match the coax-fed incident waves to the waveguide output port. The dielectric level is measured at the junction of the coax and the waveguide (see Fig. 1). Figs. 6 and 7 show the measured and simulated curves of $\left|S_{11}\right|$ at $85 \mathrm{MHz}$ as a function of the tuning distance $\left(l_{s}\right)$ for two different coupling probe radii.

The comparisons between our model and the measurements show good agreement, especially in the optimal region where the power coupling efficiency $\left(1-\left|S_{11}\right|^{2}\right)$ for the coupler can be as high as 95 percent at $85 \mathrm{MHz}$. The measurements also show that, for different coupling probe radii, the minimum reflection point ( $\left|S_{11}\right|_{\min }$ ) may change slightly. This may be due to the reactance's dependence on the probe radius as seen in (2b) and the slightly different current distributions around the probe surface caused by the existing boundary (waveguide side walls).

A small interface offset in the dielectric level is also included in our simulation $(1.5 \mathrm{~cm}$ into the coax for our case) because the adjustment of the sliding-short results in a small shift in the fluid dielectric level into the coax transition. In addition, the minima of $\left|S_{11}\right|$ for the mea- sured results did not exactly coincide with those from the theoretical model. The tuning offset, which is the difference between the two minima, is used to compensate for this discrepancy and is given as $\Delta l_{s} / \lambda_{g 10}$ in the associated figures. This small offset is most likely due to approximations made in our theoretical model (e.g., a sinusoidal probe current distribution, small probe radius-to-waveguide width ratio, etc.).

This waveguide coupler is designed for high power coupling; therefore, large coax and probes are used as indicated in Figs. 6 and 7. These large probes probably cause small errors in the model because of our previous assumptions of an azimuthal symmetry of the probe's surface current and the small probe radius-to-waveguide width ratio $(r / a)$. Also, since we desire high power coupling, an optimal operation region will always be required to minimize $\left|S_{11}\right|$ and maximize the power coupling efficiency. Hence, when used for high power applications, the waveguide system must be tuned for operation in the optimal region as seen in Figs. 6 and 7 to maintain maximum power coupling.

\section{Loaded Waveguide Coupler}

Our waveguide coupler was built for laboratory tests in the ICRF. An appropriate, large ICRF plasma test facility is not available and would be quite expensive for our plasma loading measurements. From the point of view of the loading reflection coefficient and the normalized loading impedance, however, our waveguide coupler can still be tested for its response to a plasma load. To accomplish this, we provide the coupler with a simulated load which presents a loading reflection coefficient (i.e., the same normalized loading impedance) comparable to that reflection coefficient which we would obtain from our computational model for plasma coupling [8]. This equivalent load is called simulated plasma loading. The plasma load simulation is feasible in the transmission line model and can be achieved in terms of an impedance transformation for a given plasma surface impedance or surface reflection coefficient. Calculations by Lam et al. [13] for the ICRF plasma reflection with respect to this waveguide coupler (and a parabolic or Gaussian plasma density profile) have shown a range of plasma surface reflection coefficients, $\Gamma_{p}$, and plasma surface impedances, $Z_{p}$. Values representative of these results have been chosen to be simulated.

The following sections describe how to experimentally simulate a plasma load in our waveguide coupler and provide comparisons between the measurements and our model for $S_{11}^{\prime}(3)$ with a loaded waveguide coupler.

\section{A. Simulated Plasma Load}

The simulation of a plasma load is illustrated in Fig. 8, which is a side view of the waveguide coupler. An inductive diaphragm is placed at plane 3 and has good contact with the waveguide walls. The parallel, normalized inductance $\left(X / Z_{w}\right)$ of this diaphragm can be determined by the geometrical parameters such as the diaphragm-to- 


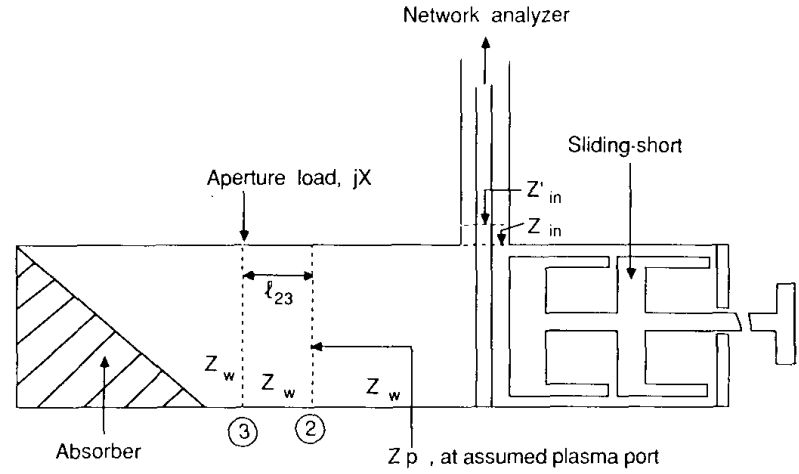

Fig. 8. Simulation of the plasma impedance, $Z_{p}$, in a waveguide.

waveguide width ratio $\left(d^{\prime} / a\right)$ and the normalized wavelength $(\lambda / a)[14]$. This diaphragm inductance is transformed back to plane 2 , where the assumed plasma port is located. If we denote $y_{3}$ as the normalized diaphragm admittance at plane 3 combined with the waveguide admittance

$$
y_{3}=\frac{-j B}{Y_{w}}+1=\frac{Z_{w}}{j X}+1
$$

where $Z_{w}\left(Y_{w}\right)$ is the waveguide characteristic impedance (admittance), we obtain a transformed, normalized plasma admittance $y_{p}$ at plane 2

$$
y_{p}=\frac{y_{3}+j \tan \left(\beta_{10} l_{23}\right)}{1+j y_{3} \tan \left(\beta_{10} l_{23}\right)}
$$

where $\beta_{10}$ is the $T E_{10}$ mode propagation constant and $l_{23}$ is the distance between plane 2 and plane 3 . With appropriate designs for the diaphragm size, the normalized operation frequency, and the transformation distance $l_{23}$, we are able to present a normalized plasma impedance $Z_{p} / Z_{w}$ at plane 3 , the plasma port.

Numerical analyses have been made and laboratory measurements have been conducted for some plasma impedance values in order to investigate the accuracy of our plasma impedance simulations. Tests were made over both the RF and the ICRF bands for an air-filled and a dielectric-filled $\left(\epsilon_{r}=78\right)$ waveguide, respectively. Note that, although we consider different dielectric fillers in the waveguide, the formula we used to calculate the diaphragm inductance always gives the same normalized values [14]. Therefore, the simulation model is valid independent of the dielectric placed in the waveguide coupler.

The time-domain gating function was again applied to measure the normalized plasma impedance. The measured frequency response of $y_{p}$ is plotted on a Smith chart (dashed lines) and compared with our model as shown in Fig. 9. The measurement frequency ranges from $f / f_{c}=$ 1.24 to 1.65 with the $f_{c}$ of the $T E_{10}$ cutoff frequency being $68 \mathrm{MHz}$ for ICRF and $606 \mathrm{MHz}$ for RF. Comparisons of the two curves in Fig. 9 show a fairly close agreement between our simulated plasma impedances and those measured. In the mid-band ranges the correspondence is even better. (The errors between the measurements and the simulations are mainly due to the diaphragm's inductance calculation and the truncation effect [11], [12] imposed by the time-domain gating function of the network analyzer.) Note that the curves for $y_{p}$ cover both the inductive and the capacitive regions. That is, our simulation model is valid for either an inductive or a capacitive reactance plasma surface impedance/admittance.

\section{B. Input Reflection Coefficient and Power Coupling Efficiency}

We next proceeded to examine the interaction of the dielectric-filled waveguide coupler with a simulated plasma load utilizing our technique of simulating plasma loads in a waveguide. The input reflection coefficient is measured at port 1 and the simulated plasma is set up as in Fig. 8. Here the effect of the distance between the coupling probe and the plasma port, $l$, is included as shown in (3) and (4).

We used the HP 8510 network analyzer for the $\left|S_{11}^{\prime}\right|$ measurements at $85 \mathrm{MHz}$ with a copper probe and a probe radius of $1.65 \mathrm{~cm}$. The two cases of plasma load considered were $Z_{p}=8.6+j 45 \Omega$ and $Z_{p}=17+j 19 \Omega$. Figs. 10 and 11 show the measurement's and our model's values of $\left|S_{11}^{\prime}\right|$ for two plasma loaded cases as functions of the tuning distance $\left(l_{s}\right)$. Both figures show a good prediction of $l_{s}$ for the low $\left|S_{11}^{\prime}\right|$ region as indicated by the small $\Delta l_{s}$ offset percentage.

For $Z_{p}=8.6+j 45 \Omega$, the input reflection coefficient $\left|S_{11}^{\prime}\right|$ can be as low as 0.4 (VSWR $=2.3$ ) and the associated power coupling efficiency is approximately 84 percent with an optimal setting of the tuning distance, $l_{s}$. For Fig. 11 , where $Z_{p}=17+j 19 \Omega$, the measured minimum $\left|S_{11}^{\prime}\right|$ is 0.5 (VSWR $=3.0$ ) and this yields an optimal power coupling efficiency of 75 percent. Note that the optimal power coupling efficiencies for the loaded coupler are uniformly smaller than those for the matched coupler when comparing Figs. 10 and 11 and Figs. 6 and 7. This is reasonable since the loaded waveguide coupler is more difficult to match by sliding-short tuning. Discrepancies between the reflection measurements and the models always exist for both matched and loaded couplers. With the loaded waveguide coupler, the differences are larger in the optimal region and smaller in the detuned region, as seen in Figs. 10 and 11. These errors also change with the values of $Z_{p}$ used for the plasma loads.

\section{Conclusions}

We have measured the attenuation due to the deionized water used for our waveguide dielectric filler. The average $Q$ values and the measured attenuation level $(0.13$ $\mathrm{dB} / \mathrm{ft}$ ) ensure that deionized water has low losses in the ICRF. These measurements allow us to use deionized water as a low cost, suitable dielectric with a high permittivity for our waveguide measurements. With this fluid dielectric, we can take full advantage of the adjustable 


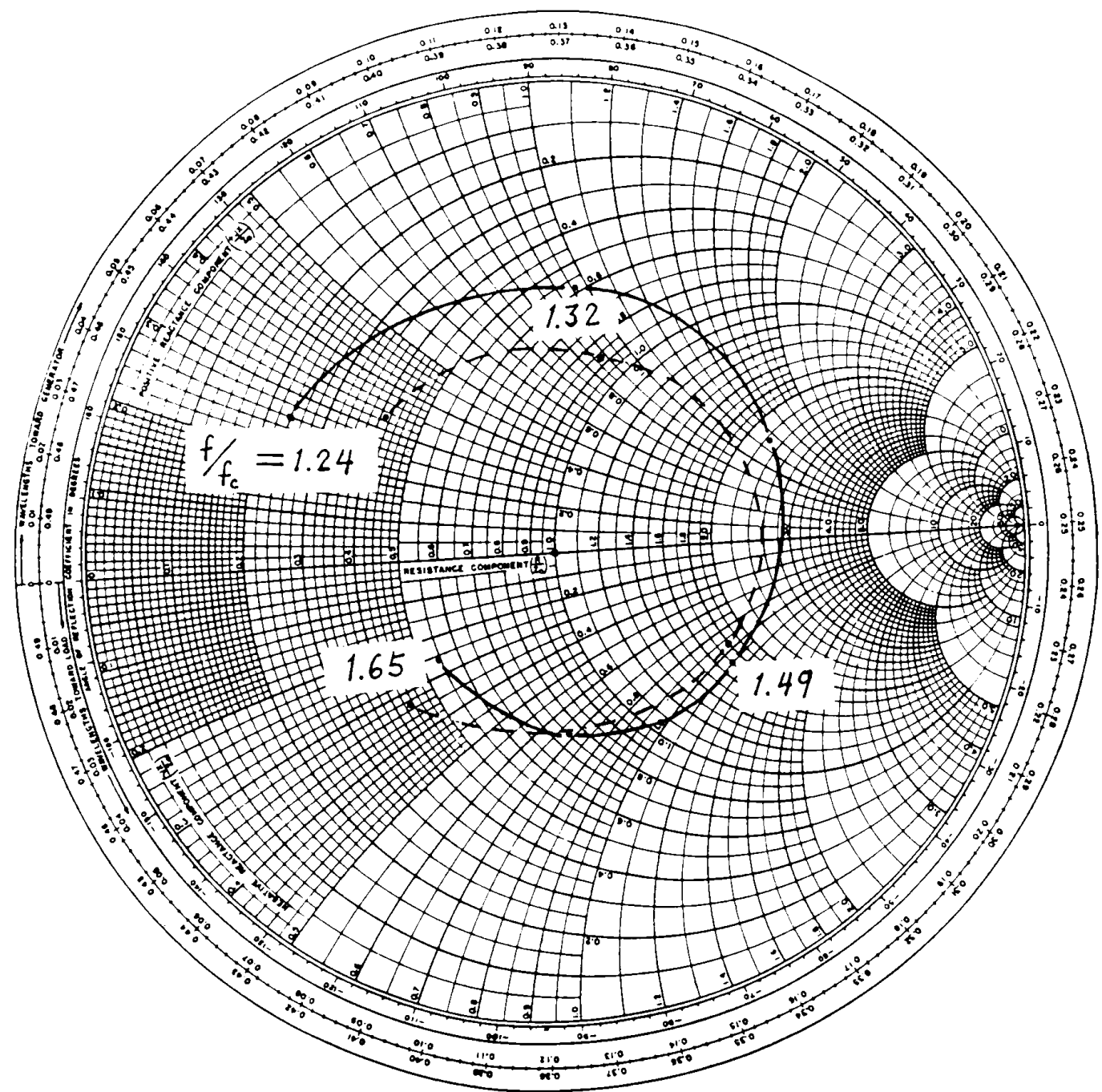

Fig. 9. Comparison of the measurement (dashed line) and the calculation (solid line) of a plasma impedance. The cutoff frequency, $f_{c}$, is $606 \mathrm{MHz}$ and $68 \mathrm{MHz}$ for the air-filled and dielectric-filled waveguides, respectively.

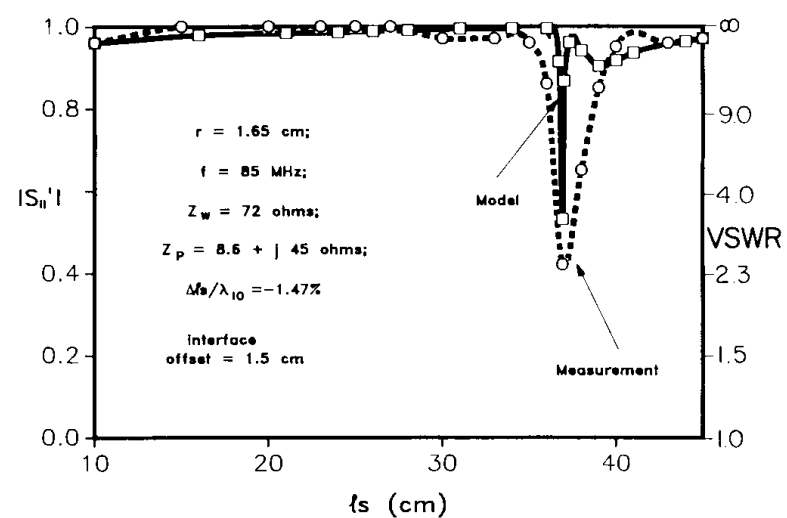

Fig. 10. Field reflection versus the tuning distance for a loaded waveguide coupler. The relative dielectric permittivity is 78 and the probe radius is $1.65 \mathrm{~cm}$.

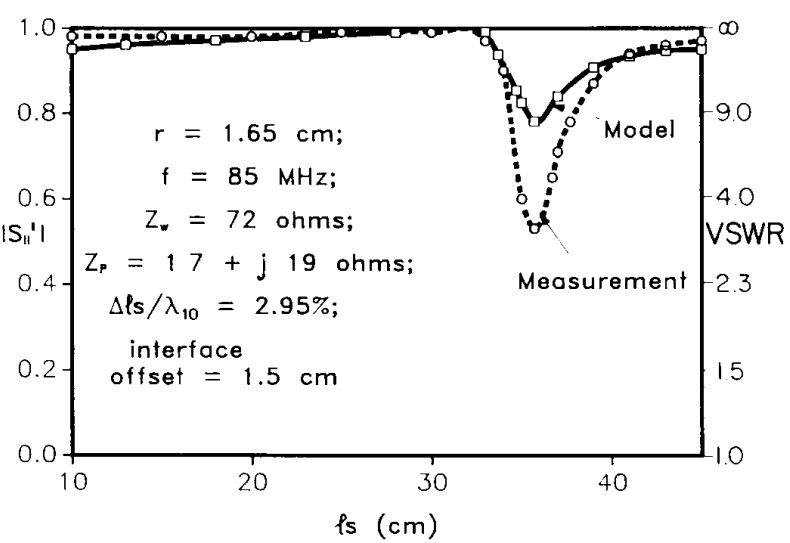

Fig. 11. Field reflection versus the tuning distance for a loaded waveguide coupler. The relative dielectric permittivity is 78 and the probe radius is $1.65 \mathrm{~cm}$. 
sliding-short for tuning and matching the waveguide coupler to plasma loads.

We have also developed a model for plasma load simulation in laboratory tests. Using a HP 8510 network analyzer and its time-domain gating function, we obtain reasonable accuracy for this simulation and are able to apply this technique to the measurements of the loaded coupler. The tests for the phase shift, $\phi_{12}$, required for the above reflection and coupling measurements show a very small deviation from our model (4).

Measurements have been carried out to experimentally verify our waveguide coupler models. We have found good agreement between our measurements and coupling models for both the matched and loaded dielectric-filled waveguides in the ICRF $(\simeq 85 \mathrm{MHz})$. For a matched coupler, we obtain an optimal power-coupling efficiency of 95 percent and for a simulated-plasma loaded coupler this value can be in the range of 80 percent, depending upon the plasma load. If it is assumed that the effects of the high neutron flux present in a reactor environment on the dielectric strength of water are negligible and that the dielectric breakdown strength of water is approximately 3 $\mathrm{kV} / \mathrm{cm}$ at nearly 80 percent coupling efficiency, we estimate the power handling capability of the water-filled coupler to be in the range of 3-6 MW.

One might also consider a dielectric-filled ICRF waveguide coupler at moderate power levels to test its coupling properties compared to coil launchers. Ultimately, a vacuum waveguide launcher, perhaps of the folded-guide type [15], might offer a viable and preferable alternative to a coil antenna launcher in a high neutron and plasma flux tokamak reactor environment.

\section{REFERENCES}

[1] J. Jacquinot et al., "ICRF studies on JET," Plasma Physics and Controlled Fusion, vol. 28, no. 1A, pp. 1-15, 1986.

[2] W. L. Gardner et al., "ICH antenna development on the ORNL RF test facility," Bull. Amer. Phys. Soc., vol. 31, p. 1418, Oct. 1986.

[3] C. M. Fortgang and D. Q. Hwang, "Measurements of the electrostatic and electromagnetic fields of Faraday shielded half-turn loop type ICRF antennas, " Princeton Plasma Physics Lab., Princeton, NJ, Rep. PPPL-2183, Dec. 1984.

[4] F. W. Perkins and R. F. Kluge, " A resonant cavity ICRF coupler for large tokamaks," IEEE Trans. Plasma Sci., vol. PS-12, pp. 161172 , June 1984.

[5] H. Arai and H. Goto, "A cutoff waveguide ICRF coupler for large tokamaks.” IEEE Trans. Plasma Sci., vol. PS-13, June 1985.

[6] O. C. Eldridge, D. J. Hoffman, and P. J. Kortman, "Status and plans for U.S. and international radio-frequency experiments on fusion devices," Fusion Tech., vol. 6, Sept. 1984.

[7] S. Knowlton et al., "Energy confinement of lower-hybrid-currentdriven tokamak plasmas," Phys. Rev. Lett., vol. 57, pp. 587-590, Aug. 1986.

[8] N. Lam, J. L. Lee, J. Scharer, and R. Vernon, "Analysis of dielectric-filled waveguide coupling to plasma in the ICRF," IEEE Trans. Plasma Sci., vol. PS-14, pp. 271-276, June 1986.

[9] J. M. Jarem, "A multifilament method-of-moments solution for the input impedance of a probe-excited semi-infinite waveguide," IEEE Trans. Microwave Theory Tech., vol. MTT-35, Jan. 1987.

[10] J. D. Jackson, Classical Electrodynamics. New York: Wiley, 1975, p. 292.

[11] Network Analyzer Operating and Programming Manual, HewlettPackard Co., Santa Rosa, CA, HP8510, 1984, pp. 145-148.

[12] "Vector measurements of high frequency networks" in HewlettPackard Seminar Notes, Hewlett-Packard, Palo Alto, CA, Mar. 1987, pp. 4-1-4-30, A-5.

[13] N. T. Lam, E. J. Sigal, O. C. Eldridge, and J. Scharer, "Coupling to tokamaks with divertors for various launcher geometries," Bull. Amer. Phys. Soc., vol. 32, p. 1951, Oct. 1987.

[14] N. Marcuvitz, Waveguide Handbook. London: Peregrinus, 1986, pp. 224-227.

[15] T. L. Owens, "A folded waveguide coupler for plasma heating in the ICRF," IEEE Trans. Plasma Sci., vol. PS-14, Dec. 1986. 